\title{
GENERALIZED WINTGEN INEQUALITY FOR SUBMANIFOLDS IN KENMOTSU SPACE FORMS
}

\author{
MOHD. AQUIB AND MOHAMMAD HASAN SHAHID
}

\begin{abstract}
In this paper, we obtain the generalized Wintgen inequality for Legendrian submanifolds in Kenmotsu space forms and discuss the equality case of the inequality. Further, we discuss the inequality for bi-slant submanifolds in the same ambient space and derive its applications in various slant cases.
\end{abstract}

\section{Introduction}

Kenmotsu manifold is an important class of manifolds endowed with geometrical structure. K. Kenmotsu [6], introduced and studied these manifolds. Afterward, many geometers studied the geometry of submanifolds in Kenmotsu manifolds due to its rich geometric importance $[1,7,10]$.

On the other hand, the Wintgen inequality is a sharp geometric inequality for surfaces in 4-dimensional Euclidean space involving Gauss curvature (intrinsic invariants), normal curvature and square mean curvature (extrinsic invariants).

P. Wintgen [11], proved that the Gauss curvature $\mathscr{K}$, the normal curvature $\mathscr{K}^{\perp}$ and the squared mean curvature $\|\mathscr{H}\|^{2}$ for any surface $M^{2}$ in $E^{4}$ satisfy the following inequality:

$$
\|\mathscr{H}\|^{2} \geq \mathscr{K}+\left|\mathscr{K}^{\perp}\right|
$$

and the equality holds if and only if the ellipse of curvature of $M^{2}$ in $E^{4}$ is a circle.

Later, it was extended by I. V. Gaudalupe et.al. [4] for arbitrary codimension $m$ in real space forms $\bar{M}^{m+2}(c)$ as

$$
\|\mathscr{H}\|^{2}+c \geq \mathscr{K}+\left|\mathscr{K}^{\perp}\right| .
$$

They also discussed the equality case of the inequality.

Received May 15, 2018, accepted September 17, 2018.

2010 Mathematics Subject Classification. Primary 53B05; Secondary 53B20, 53C40.

Key words and phrases. Wintgen inequality, Legendrian submanifold, Kenmotsu space forms, bi-slant submanifold.

Corresponding author: Mohd. Aquib. 
In 1999, De Smet, Dillen, Verstraelen and Vrancken conjectured the generalized Wintgen inequality for submanifolds in real space form. This conjecture is also known as DDVV conjecture. It was proved by Ge and Tang [3]. Recently, the DDVV inequality has been obtained by distinct researchers for different submanifolds and different ambient manifolds [2, 9].

In present article, we obtain the generalized Wintgen inequalities for Legendrian submanifolds in Kenmotsu space forms. We also discuss such inequality for various slant cases as an application of the inequality obtained.

\section{Submanifolds in Kenmotsu space forms}

An odd $(2 m+1)$-dimensional smooth manifold $\bar{M}$ is called a Kenmotsu manifold, if it admits an endomorphism $\varphi$ of its tangent bundle $T \bar{M}$, a structure vector field $\xi$, and a 1-form $\eta$ satisfying the following:

$$
\begin{gathered}
\varphi^{2}=-I+\eta \oplus \xi, \quad \eta(\xi)=1, \quad \eta \circ \phi=0, \\
g(\varphi X, \varphi Y)=g(X, Y)-\eta(X) \eta(Y), \quad \eta(X)=g(X, \xi), \\
\left(\bar{\nabla}_{X} \varphi\right) Y=g(\varphi X, Y)-\eta(Y) \varphi X \\
\bar{\nabla}_{X} \xi=X-\eta(X) \xi
\end{gathered}
$$

for any $X, Y$ tangent to $\bar{M}[6]$.

A Kenmotsu manifold $\bar{M}$ with constant $\phi$-sectional curvature $c$ is called a Kenmotsu space forms and is denoted by $\bar{M}(c)$. The curvature tensor $\bar{R}$ for Kenmotsu space forms $\bar{M}(c)$ is given by

$$
\begin{aligned}
\bar{R}(X, Y, Z, W)= & \frac{c-3}{4}\{g(Y, Z) g(X, W)-g(X, Z) g(Y, W)\} \\
& +\frac{c+1}{4}\{g(X, \phi W) g(Y, \phi Z)-g(X, \phi Z) g(Y, \phi W) \\
& -2 g(X, \phi Y) g(Z, \phi W)-g(X, W) \eta(Y) \eta(Z) \\
& +g(X, Z) \eta(Y) \eta(W)-g(Y, Z) \eta(X) \eta(W) \\
& +g(Y, W) \eta(X) \eta(Z)\},
\end{aligned}
$$

for all $X, Y, Z \in T \bar{M}$.

Let $M$ be a submanifold of an almost contact metric manifold $\bar{M}$ with induced metric $g$; if $\nabla$ and $\nabla^{\perp}$ are the induced connections on the tangent bundle $T M$ and the normal bundle $T^{\perp} M$ of $M$, respectively, then the Gauss and Weingarten formulas are given by

$$
\begin{aligned}
& \bar{\nabla}_{X} Y=\nabla_{X} Y+h(X, Y), \\
& \bar{\nabla}_{X} N=-S_{N} X+\nabla_{X}^{\perp} N,
\end{aligned}
$$


for vector fields $X, Y \in T M$ and $N \in T^{\perp} M$, where $h, S_{N}$ and $\nabla^{\perp}$ is the second fundamental form, the shape operator and the normal connection respectively and for the immersion of $M$ into $\bar{M}$ the second fundamental form and the shape operator are related by the following equation

$$
g(h(X, Y), N)=g\left(S_{N} X, Y\right),
$$

for vector fields $X, Y \in T M$ and $N \in T^{\perp} M$.

The equation of Gauss is given by

$$
\bar{R}(X, Y, Z, W)=R(X, Y, Z, W)+g(h(X, Z), h(Y, W))-g(h(X, W), h(Y, Z)),
$$

for $X, Y, Z, W \in T M$, where $\bar{R}$ and $R$ represent the curvature tensor of $\bar{M}(c)$ and $M$ respectively and the Ricci equation is given

$$
R^{\perp}(X, Y, \xi, \eta)=\frac{c+1}{4}[g(J X, \xi) g(J Y, \eta)-g(J X, \eta) g(J Y, \xi)]-g\left(\left[S_{\xi}, S_{\eta}\right] X, Y\right),
$$

for $X, Y \in T M$ and $\xi, \eta \in T^{\perp} M$.

The squared norm of $P$ at $p \in M$ is defined as

$$
\|P\|^{2}=\sum_{i, j=1}^{n} g^{2}\left(\varphi e_{i}, e_{j}\right)
$$

where $\left\{e_{1}, \ldots, e_{n}\right\}$ is any orthonormal basis of the tangent space $T M$ of $M$. Let $\left\{e_{1}, \ldots, e_{n}\right\}$ and $\left\{e_{n+1}, \ldots, e_{2 m+1}\right\}$ be tangent orthonormal frame and normal orthonormal frame, respectively, on $M$. The mean curvature vector field is given by

$$
\mathscr{H}=\frac{1}{n} \sum_{i=1}^{n} h\left(e_{i}, e_{i}\right)
$$

We also set

$$
\|h\|^{2}=\sum_{i, j=1}^{n} g\left(h\left(e_{i}, e_{j}\right), h\left(e_{i}, e_{j}\right)\right) .
$$

\section{Generalized Wintgen inequality}

We denote by $\mathscr{K}$ and $R^{\perp}$ the sectional curvature function and the normal curvature tensor on $M$, respectively. Then the normalized scalar curvature $\rho$ is given by [9]

$$
\rho=\frac{2 \tau}{n(n-1)}=\frac{2}{n(n-1)} \sum_{1 \leq i<j \leq n} \not{K}\left(e_{i} \wedge e_{j}\right),
$$


where $\tau$ is scalar curvature, and the normalized normal scalar curvature by [9]

$$
\rho^{\perp}=\frac{2 \tau^{\perp}}{n(n-1)}=\frac{2}{n(n-1)} \sqrt{\sum_{1 \leq i<j \leq n} \sum_{1 \leq \alpha<\beta \leq 2 m+1}\left(R^{\perp}\left(e_{i}, e_{j}, \xi_{\alpha}, \xi_{\beta}\right)\right)^{2}} .
$$

Following [12] we put

$$
\mathscr{K}_{N}=\frac{1}{4} \sum_{r, s=1}^{2 m-n+1} \operatorname{Trace}\left[S_{r}, S_{s}\right]^{2}
$$

and call it the scalar normal curvature of $M$. The normalized scalar normal curvature is given by [9] $\rho_{N}=\frac{2}{n(n-1)} \sqrt{\mathscr{K}_{N}}$.

Obviously

$$
\begin{aligned}
\mathscr{K}_{N} & =\frac{1}{2} \sum_{1 \leq r<s \leq 2 m-n+1} \operatorname{Trace}\left[S_{r}, S_{s}\right]^{2} \\
& =\sum_{1 \leq r<s \leq 2 m-n+1} \sum_{1 \leq i<j \leq n} g\left(\left[S_{r}, S_{s}\right] e_{i} \cdot e_{j}\right)^{2},
\end{aligned}
$$

for $i, j \in\{1, \ldots, n\}$ and $r, s \in\{1, \ldots, 2 m-n+1\}$.

In term of the components of the second fundamental form, we can express $\mathscr{K}_{N}$ by the formula [9]

$$
\mathscr{K}_{N}=\sum_{1 \leq r<s \leq 2 m-n+1} \sum_{1 \leq i<j \leq n}\left(\sum_{k=1}^{n} h_{j k}^{r} h_{i k}^{s}-h_{j k}^{r} h_{i k}^{s}\right)^{2}
$$

A submanifold $M$ of a Kenmotsu manifold $\bar{M}$ is said to be C-totally real submanifold if $\phi$ maps each tangent space of $M$ into the normal space, i.e. $\phi(T M) \subset T^{\perp} M$. In particular, if $n=m$, then $M$ is called Legendrian submanifold.

First we proof the following lemma, which will be required in the proof of main result.

Lemma 3.1. Let $M$ be a $n$-dimensional $C$-totally real submanifolds of a $(2 m+1)$-dimensional Kenmotsu space forms $\bar{M}(c)$. Then we have

$$
\rho_{N}+\rho \leq \frac{c-3}{4}+\frac{c+1}{2 n}+\|\not{H}\|^{2},
$$

and the equality holds if and only if the shape operator S of $M$ in $\bar{M}(c)$ takes the following form

$$
S_{e_{n+1}}=\left(\begin{array}{ccccc}
\gamma_{1} & v & 0 & \ldots & 0 \\
v & \gamma_{1} & 0 & \ldots & 0 \\
0 & 0 & \gamma_{1} & \ldots & 0 \\
\vdots & \vdots & \vdots & \ddots & \vdots \\
0 & 0 & 0 & \ldots & \gamma_{1}
\end{array}\right),
$$




$$
\begin{aligned}
S_{e_{n+2}} & =\left(\begin{array}{ccccc}
\gamma_{2}+v & 0 & 0 & \ldots & 0 \\
0 & \gamma_{2}-v & 0 & \ldots & 0 \\
0 & 0 & \gamma_{2} & \ldots & 0 \\
\vdots & \vdots & \vdots & \ddots & \vdots \\
0 & 0 & 0 & \ldots & \gamma_{2}
\end{array}\right), \\
S_{e_{n+3}} & \left(\begin{array}{ccccc}
\gamma_{3} & 0 & 0 & \ldots & 0 \\
0 & \gamma_{3} & 0 & \ldots & 0 \\
0 & 0 & \gamma_{3} & \ldots & 0 \\
\vdots & \vdots & \vdots & \ddots & \vdots \\
0 & 0 & 0 & \ldots & \gamma_{3}
\end{array}\right), \quad S_{e_{n+4}}=\ldots=S_{e_{2 m}}=S_{e_{2 m+1}}=0,
\end{aligned}
$$

where $\gamma_{1}, \gamma_{2}, \gamma_{3}$ and $v$ are real functions on $M$.

Proof. We know that

$$
\begin{aligned}
n^{2}\|\not \mathcal{H}\|^{2} & =\sum_{r=}^{2 m-n+1}\left(\sum_{i=1}^{n} h_{i i}^{r}\right)^{2} \\
& =\frac{1}{n-1} \sum_{r=1}^{2 m-n+1} \sum_{1 \leq i<j \leq n}\left(h_{i i}^{r}-h_{j j}^{r}\right)^{2}+\frac{2 n}{n-1} \sum_{r=1}^{2 m-n+1} \sum_{1 \leq i<j \leq n} h_{i i}^{r} h_{j j}^{r} .
\end{aligned}
$$

Further, from [8] we have

$$
\begin{aligned}
& \sum_{r=1}^{2 m-n+1} \sum_{1 \leq i<j \leq n}\left(h_{i i}^{r}-h_{j j}^{r}\right)^{2}+2 n \sum_{r=1}^{2 m-n+1} \sum_{1 \leq i<j \leq n}\left(h_{i j}^{r}\right)^{2} \\
& \quad \geq 2 n\left[\sum_{1 \leq r<s \leq 2 m-n+1} \sum_{1 \leq i<j \leq n}\left(\sum_{k=1}^{n}\left(h_{j k}^{r} h_{i k}^{s}-h_{i k}^{r} h_{j k}^{s}\right)\right)^{2}\right]^{\frac{1}{2}} .
\end{aligned}
$$

Now, combining (3.5), (3.7) and (3.8), we find

$$
n^{2}\|\mathscr{H}\|^{2}-n^{2} \rho_{N} \geq \frac{2 n}{n-1} \sum_{r=1}^{2 m-n+1} \sum_{1 \leq i<j \leq n}\left[h_{i i}^{r} h_{j j}^{r}-\left(h_{i j}^{r}\right)^{2}\right] .
$$

Also, from equation (2.6), we get

$$
\tau=\frac{c-3}{8} n(n-1)+\frac{c+1}{8}\{2(n-1)\}+\sum_{r=n+1}^{2 m+1} \sum_{1 \leq i<j \leq n}\left[h_{i i}^{r} h_{j j}^{r}-\left(h_{i j}^{r}\right)^{2}\right] .
$$

Using (3.1) and (3.10) in (3.9), we have the required result.

Equality case holds if and only if shape operator takes the above stated forms.

Remark 3.2. If the equality holds in the above inequality (3.6) then $M$ is called a Wintgen ideal submanifold (see [5]).

Now, we prove the following: 
Theorem 3.3. Let $M$ be a Legendrian submanifold of a Kenmotsu space forms $\bar{M}(c)$. Then

$$
\begin{aligned}
\left(\rho^{\perp}\right)^{2} \leq & {\left[\|\mathscr{H}\|^{2}-\left(\rho-\frac{c-3}{4}\right)+\frac{c+1}{2 n}\right]^{2}+\frac{2}{n(n-1)}\left(\frac{c+1}{4}\right)^{2} } \\
& +\frac{c+1}{n^{2}(n-1)^{2}}\left[n(n-1)\left(\rho-\frac{c-3}{4}\right)+\frac{c+1}{2}(n-1)\right],
\end{aligned}
$$

Proof. Let $M$ be a Legendrian submanifold of a Kenmotsu space forms $\bar{M}(c)$. We choose $\left\{e_{1}, \ldots, e_{n}, e_{n+1}=\xi\right\}$ and $\left\{e_{n+2}, \ldots, e_{2 m+1}\right\}$ as orthonormal frame and orthonormal normal frame on $M$ respectively. Putting $X=W=e_{i}, Y=Z=e_{j}, i \neq j$ from (2.5), we have

$$
\begin{aligned}
\bar{R}\left(e_{i}, e_{j}, e_{j}, e_{i}\right)= & \frac{c-3}{4}\left\{g\left(e_{j}, e_{j}\right) g\left(e_{i}, e_{i}\right)-g\left(e_{i}, e_{j}\right) g\left(e_{j}, e_{i}\right)\right\} \\
& +\frac{c+1}{4}\left\{g\left(e_{i}, \phi e_{i}\right) g\left(e_{j}, \phi e_{j}\right)-g\left(e_{i}, \phi e_{j}\right) g\left(e_{j}, \phi e_{i}\right)\right. \\
& -2 g\left(e_{i}, \phi e_{j}\right) g\left(e_{j}, \phi e_{i}\right)-g\left(e_{i}, e_{i}\right) \eta\left(e_{j}\right) \eta\left(e_{i}\right) \\
& +g\left(e_{i}, e_{j}\right) \eta\left(e_{j}\right) \eta\left(e_{i}\right)-g\left(e_{j}, e_{j}\right) \eta\left(e_{i}\right) \eta\left(e_{i}\right) \\
& \left.+g\left(e_{j}, e_{i}\right) \eta\left(e_{i}\right) \eta\left(e_{j}\right)\right\} .
\end{aligned}
$$

Combining equations (2.6) and (3.12), we obtain

$$
\begin{aligned}
R\left(e_{i}, e_{j}, e_{j}, e_{i}\right)= & \frac{c-3}{4}\left\{g\left(e_{j}, e_{j}\right) g\left(e_{i}, e_{i}\right)-g\left(e_{i}, e_{j}\right) g\left(e_{j}, e_{i}\right)\right\} \\
& +\frac{c+1}{4}\left\{g\left(e_{i}, \phi e_{i}\right) g\left(e_{j}, \phi e_{j}\right)-g\left(e_{i}, \phi e_{j}\right) g\left(e_{j}, \phi e_{i}\right)\right. \\
& -2 g\left(e_{i}, \phi e_{j}\right) g\left(e_{j}, \phi e_{i}\right)-g\left(e_{i}, e_{i}\right) \eta\left(e_{j}\right) \eta\left(e_{j}\right) \\
& +g\left(e_{i}, e_{j}\right) \eta\left(e_{j}\right) \eta\left(e_{i}\right)-g\left(e_{j}, e_{j}\right) \eta\left(e_{i}\right) \eta\left(e_{i}\right) \\
& \left.+g\left(e_{j}, e_{i}\right) \eta\left(e_{i}\right) \eta\left(e_{j}\right)\right\}+g\left(h\left(e_{i}, e_{i}\right), h\left(e_{j}, e_{j}\right)\right) \\
& -g\left(h\left(e_{i}, e_{j}\right), h\left(e_{i}, e_{j}\right)\right) .
\end{aligned}
$$

By taking summation $1 \leq i, j \leq n$ and using (2.9), (2.10) in (3.13), we derive

$$
\begin{aligned}
2 \tau & =\sum_{i \neq j}^{n-1} R\left(e_{i}, e_{j}, e_{j}, e_{i}\right)+2 \sum_{i=1}^{n-1} R\left(e_{i}, \xi, \xi, e_{i}\right) \\
& =\sum_{i, j=1}^{n} R\left(e_{i}, e_{j}, e_{j}, e_{i}\right) \\
& =n(n-1) \frac{c-3}{4}+\frac{c+1}{4}\{2(1-n)\}+n^{2}\|\not{H}\|^{2}-\|h\|^{2} .
\end{aligned}
$$

Using (3.1) in (3.14), we get

$$
\rho=\frac{c-3}{4}+\frac{c+1}{2 n}+\frac{n^{2}\|\not{H}\|^{2}}{n(n-1)}-\frac{\|h\|^{2}}{n(n-1)},
$$


which implies

$$
n^{2}\|\mathscr{H}\|^{2}-\|h\|^{2}=n(n-1)\left(\rho-\frac{c-3}{4}\right)-\frac{c+1}{4}\{2(1-n)\} .
$$

Further, equation (2.7) implies

$$
R^{\perp}\left(e_{i}, e_{j}, \xi_{r}, \xi_{s}\right)=\frac{c+1}{4}\left\{-\left(\delta_{i r} \delta_{j s}-\delta_{j r} \delta_{i s}\right)\right\}+g\left(\left[S_{\xi_{r}}, S_{\xi_{s}}\right] e_{i}, e_{j}\right)
$$

for all $i, j \in\{1, \ldots, n\}$ and $r, s \in\{1, \ldots, n\}$.

Then we have

$$
\begin{aligned}
\left(\tau^{\perp}\right)^{2} & =\left(R^{\perp}\left(e_{i}, e_{j}, \xi_{r}, \xi_{s}\right)\right)^{2} \\
& =\left[\frac{c+1}{4}\left(\delta_{i r} \delta_{j s}-\delta_{j r} \delta_{i s}\right)-g\left(\left[S_{\xi_{r}}, S_{\xi_{s}}\right] e_{i}, e_{j}\right)\right]^{2} \\
& =\frac{n(n-1)}{2}\left(\frac{c+1}{4}\right)^{2}+\frac{n^{2}(n-1)^{2}}{4} \rho_{N}^{2}-\frac{c+1}{4}\|h\|^{2}+\frac{c+1}{4} n^{2}\|\mathscr{H}\|^{2} .
\end{aligned}
$$

Above equation can be re-written as

$$
\left(\rho^{\perp}\right)^{2}=\frac{2}{n(n-1)}\left(\frac{c+1}{4}\right)^{2}+\rho_{N}^{2}-\frac{c+1}{n^{2}(n-1)^{2}}\|h\|^{2}+\frac{c+1}{(n-1)^{2}}\|\mathscr{H}\|^{2} .
$$

Now, from (3.16) and (3.19), we have

$$
\left(\rho^{\perp}\right)^{2}=\frac{2}{n(n-1)}\left(\frac{c+1}{4}\right)^{2}+\rho_{N}^{2}+\frac{c+1}{n^{2}(n-1)^{2}}\left[n(n-1)\left(\rho-\frac{c-3}{4}\right)+\frac{c+1}{2}(n-1)\right] .
$$

Taking into account (3.6) and (3.20), we obtain the required inequality. Further, if the shape operator takes the forms as in Lemma 3.1, then the equality of the inequality holds. Hence, from [5] we have our assertion.

\section{4. bi-slant submanifolds in Kenmotsu manifold}

A submanifold $M$ in an almost contact metric manifold $\bar{M}$ is said to be slant if for any $p \in M$ and any $X \in T_{p} M$, linearly independent on $\xi$, the angle between $\phi X$ and $T_{p} M$ is a constant $\theta \in\left[0, \frac{\pi}{2}\right]$, called the slant angle of $M$ in $\bar{M}$.

A submanifold $M$ of an almost contact metric manifold $\bar{M}$ is said to be a bi-slant submanifold, if there exist two orthogonal distributions $D_{1}$ and $D_{2}$, such that

1. $T M$ admits the orthogonal direct decomposition, i.e., $T M=D_{1} \oplus D_{2} \oplus\langle\xi\rangle$.

2. $D_{i}$ is the slant distribution with slant angle $\theta_{i}$ for any $i=1,2$. 
Table 1: Definition.

\begin{tabular}{|c|l|l|l|l|c|c|}
\hline S.N. & $\bar{M}$ & \multicolumn{1}{|c|}{$M$} & \multicolumn{1}{c|}{$D_{1}$} & \multicolumn{1}{c|}{$D_{2}$} & $\theta_{1}$ & $\theta_{2}$ \\
\hline$(1)$ & $\bar{M}$ & bi-slant & slant & slant & slant angle & slant angle \\
\hline$(2)$ & $\bar{M}$ & semi-slant & invariant & slant & 0 & slant angle \\
\hline$(3)$ & $\bar{M}$ & hemi-slant & slant & anti-invariant & slant angle & $\frac{\pi}{2}$ \\
\hline$(4)$ & $\bar{M}$ & CR & invariant & anti-invariant & 0 & $\frac{\pi}{2}$ \\
\hline$(5)$ & $\bar{M}$ & slant & \multicolumn{2}{l}{ either $D_{1}=0$ or $D_{2}=0$} & either $\theta_{1}=\theta_{2}=\theta$ or $\theta_{1}=\theta_{2} \neq \theta$ \\
\hline
\end{tabular}

In fact, semi-slant submanifolds, hemi-slant submanifolds, CR-submanifolds, slant submanifolds can be obtained from bi-slant submanifolds in particular. We can see the case in the following table:

Invariant and anti-invariant submanifolds are the slant submanifolds with slant angle $\theta=0$ and $\theta=\frac{\pi}{2}$ respectively and when $0<\theta<\frac{\pi}{2}$, then slant submanifold is called proper slant submanifold.

If $M$ is a bi-slant submanifold in generalized Kenmotsu space forms $\bar{M}$, then one can easily see that

$$
\|P\|^{2}=\sum_{i, j}^{n} g^{2}\left(P e_{i}, e_{j}\right)=2\left(d_{1} \cos ^{2} \theta_{1}+d_{2} \cos ^{2} \theta_{2}\right),
$$

where $\operatorname{dim} D_{1}=2 d_{1}$ and $\operatorname{dim} D_{2}=2 d_{2}$.

Now, we shall state and prove the generalized Wintgen inequality for bi-slant submanifolds in Kenmotsu space forms.

Theorem 4.1. Let $M$ be a bi-slant submanifold of a Kenmotsu space forms $\bar{M}(c)$. Then

$$
\rho_{N} \leq\|\mathscr{H}\|^{2}-\left(\rho-\frac{c-3}{4}\right)-\frac{c+1}{2 n}+\frac{3(c+1)}{2 n(n-1)}\left(d_{1} \cos ^{2} \theta_{1}+d_{2} \cos ^{2} \theta_{2}\right) .
$$

Proof. Let $M$ be a bi-slant submanifold of a Kenmotsu space forms $\bar{M}(c)$ and let $\left\{e_{1}, \ldots, e_{n-1}, e_{n}=\right.$ $\xi\}$ be an orthonormal frame on $M$ and $\left\{e_{n+1}, \ldots, e_{2 m+1}\right\}$ be normal orthonormal frame on $M$.

Equation (2.6) can be re-written as

$$
\begin{aligned}
R(X, Y, Z, W)= & \frac{c-3}{4}\{g(Y, Z) g(X, W)-g(X, Z) g(Y, W)\} \\
& +\frac{c+1}{4}\{g(X, \phi W) g(Y, \phi Z)-g(X, \phi Z) g(Y, \phi W) \\
& -2 g(X, \phi Y) g(Z, \phi W)-g(X, W) \eta(Y) \eta(Z) \\
& +g(X, Z) \eta(Y) \eta(W)-g(Y, Z) \eta(X) \eta(W)
\end{aligned}
$$




$$
\begin{aligned}
& +g(Y, W) \eta(X) \eta(Z)\}-g(h(X, Z), h(Y, W)) \\
& +g(h(X, W), h(Y, Z)),
\end{aligned}
$$

which implies

$$
\begin{aligned}
\tau= & \frac{c-3}{8} n(n-1)+\frac{c+1}{8}\left\{2(1-n)+3 g^{2}\left(\phi e_{j}, e_{i}\right)\right\}+\sum_{r=1}^{2 m-n+1} \sum_{1 \leq i<j \leq n}\left[h_{i i}^{r} h_{j j}^{r}-\left(h_{i j}^{r}\right)^{2}\right] \\
= & \frac{c-3}{8} n(n-1)-\frac{c+1}{4}(n-1)+\frac{3(c+1)}{8}\left(d_{1} \cos ^{2} \theta_{1}+d_{2} \cos ^{2} \theta_{2}\right) \\
& +\sum_{r=1}^{2 m-n+1} \sum_{1 \leq i<j \leq n}\left[h_{i i}^{r} h_{j j}^{r}-\left(h_{i j}^{r}\right)^{2}\right] .
\end{aligned}
$$

Similar as in the proof of Lemma 3.1, we get

$$
n^{2}\|\mathscr{H}\|^{2}-n^{2} \rho_{N} \geq \frac{2 n}{n-1} \sum_{r=1}^{2 m-n+1} \sum_{1 \leq i<j \leq n}\left[h_{i i}^{r} h_{j j}^{r}-\left(h_{i j}^{r}\right)^{2}\right] .
$$

Combining equations (4.4) and (4.5), we find

$$
\rho_{N} \leq\|\mathscr{H}\|^{2}-\left(\rho-\frac{c-3}{4}\right)-\frac{c+1}{2 n}+\frac{3(c+1)}{2 n(n-1)}\left(d_{1} \cos ^{2} \theta_{1}+d_{2} \cos ^{2} \theta_{2}\right) .
$$

An immediate consequence of the Theorem 4.1 yields the following.

Corollary 4.2. Let $M$ be a semi-slant submanifold of a Kenmotsu space forms $\bar{M}(c)$. Then

$$
\rho_{N} \leq\|\mathscr{H}\|^{2}-\left(\rho-\frac{c-3}{4}\right)-\frac{c+1}{2 n}+\frac{3(c+1)}{2 n(n-1)}\left(d_{1}+d_{2} \cos ^{2} \theta_{2}\right) .
$$

Corollary 4.3. Let $M$ be a hemi-slant submanifold of Kenmotsu space forms $\bar{M}(c)$. Then

$$
\rho_{N} \leq\|\mathscr{H}\|^{2}-\left(\rho-\frac{c-3}{4}\right)-\frac{c+1}{2 n}+\frac{3(c+1)}{2 n(n-1)} d_{1} \cos ^{2} \theta_{1} .
$$

Corollary 4.4. Let $M$ be a CR-submanifold of a Kenmotsu space forms $\bar{M}(c)$. Then

$$
\rho_{N} \leq\|\mathscr{H}\|^{2}-\left(\rho-\frac{c-3}{4}\right)-\frac{c+1}{2 n}+\frac{3(c+1)}{2 n(n-1)} d_{1} .
$$

Corollary 4.5. Let $M$ be a slant submanifold of a Kenmotsu space forms $\bar{M}(c)$. Then

$$
\rho_{N} \leq\|\mathscr{H}\|^{2}-\left(\rho-\frac{c-3}{4}\right)-\frac{c+1}{2 n}+\frac{3(c+1)}{4(n-1)} \cos ^{2} \theta .
$$

Corollary 4.6. Let $M$ be a invariant submanifold of a Kenmotsu space forms $\bar{M}(c)$. Then

$$
\rho_{N} \leq\|\mathscr{H}\|^{2}-\left(\rho-\frac{c-3}{4}\right)-\frac{c+1}{2 n}+\frac{3(c+1)}{4(n-1)} .
$$


Corollary 4.7. Let $M$ be a anti-invariant submanifold of a Kenmotsu space forms $\bar{M}(c)$. Then

$$
\rho_{N} \leq\|\mathscr{H}\|^{2}-\left(\rho-\frac{c-3}{4}\right)-\frac{c+1}{2 n} .
$$

Remark 4.8. The proof of the Corollary 4.2 - Corollary 4.7 is similar to the Theorem 4.1 . We obtain the proof of the Corollary 4.2 - Corollary 4.5 with the help of Table 1 and Theorem 4.1. The Corollary 4.6 and Corollary 4.7 is obtained by putting $\theta=0$ and $\theta=\frac{\pi}{2}$ in Corollary 4.5 respectively.

\section{Acknowledgement}

The authors wish to thank the referees for their many valuable and helpful suggestions in order to improve this manuscript.

\section{References}

[1] K. Arslan, I. Mihai, C. Murathan and C. Ozgur, Ricci curvature of submanifolds in Kenmotsu space forms, Int. J. Math. Math. Sci., 29 (2002), 719-726.

[2] M. N. Boyom, M. Aquib, M. H. Shahid and M. Jamali, Generalized wintegen type inequality for lagrangian submanifolds in holomorphic statistical space forms, In: Nielsen F., Barbaresco F. (eds) Geometric Science of Information, GSI 2017, Lecture Notes in Computer Science, Springer, Cham 10589.

[3] J. Ge and Z. Tang, A proof of the DDVV conjecture and its equality case, Pacific J. Math., 237(2009), (1), 87-95.

[4] I. V. Guadalupe and L. Rodriguez, Normal curvature of surfaces in space forms, Pacific J. Math., 106 (1983), 95-103.

[5] S. Haesen and L. Verstraelen, Natural Intrinsic Geometrical Symmetries, Symmetry, Integrability and Geometry: Methods and Applications, 5 (2009), paper 086, pp. 15.

[6] K. Kenmotsu, A class of almost contact Riemannian manifolds, Tohoku Math. J., 24 (1972), 93-103.

[7] C. W. Lee, J. W. Lee and G. E. Vilcu, Optimal inequalities for the normalized $\delta$-Casorati curvatures of submanifolds in Kenmotsu space forms, Adv. Geom., 17(2017), 355-362.

[8] Z. Lu, Normal scalar curvature conjecture and its applications, J. fucnt. Analysis, 261 (2011), 1284-1308.

[9] I. Mihai, On the generalized Wintgen inequality for lagrangian submanifolds in complex space form, Nonlinear Analysis, 95 (2014), 714-720.

[10] C. Murathan, K. Arslan, R. Ezentas and I. Mihai, Warped product submanifolds in Kenmotsu space forms, Taiwanese. J. Math., 10(2006), 1431-1441.

[11] P. Wintgen, Sur l'inégalité de Chen-Wilmore, C. R. Acad. Sci. Paris Ser. A-B, 288 (1979), A993-A995.

[12] K. Yano and M. Kon, Anti-invariant Submanifolds, M. Dekker, New York, 1976.

Department of Mathematics, Faculty of Natural Sciences, Jamia Millia Islamia, New Delhi-110025, India.

E-mail: aquib80@gmail.com

Department of Mathematics, Faculty of Natural Sciences, Jamia Millia Islamia, New Delhi-110025, India.

E-mail: hasan.jmi@yahoo.com 